\title{
UN ENFOQUE INNOVADOR, TRANSFORMADOR Y PROSPECTIVO DEL TRABAJO METODOLÓGICO EN LA FORMACIÓN DE PREGRADO
}

\author{
Maribel Ferrer Vicente, Dra. C. \\ Universidad de Oriente, Cuba \\ maribel@uo.edu.cu
}

\author{
María Myrna Castillo Rodríguez, Dra. C. \\ Universidad de Oriente, Cuba \\ myrnac@uo.edu.cu
}

Palabras claves: trabajo metodológico, innovación, transformación, prospectiva y formación integral del profesional.
Recibido: 17 de Octubre de 2017

Aceptado: 10 de Noviembre de 2017

\section{RESUMEN}

La gestión del proceso de formación del profesional de pregrado tiene como vía esencial un sistema de trabajo metodológico que capacite a directivos y docentes para la comprensión y puesta en práctica de los planes y programas de estudio, sustentado en el principio pedagógico de la unidad de la instrucción y la educación y de vinculación de la teoría con la práctica, que sitúa la labor educativa en el centro del proceso y sus resultados, con un enfoque innovador, transformador y prospectivo. El objetivo de este artículo es revelar cualidades que propicien la comprensión de la importancia del trabajo metodológico como soporte estratégico de la gestión del proceso de formación del profesional en las universidades, de su naturaleza didáctica, sistémica, preventiva y participativa para promover el cambio a favor del mejoramiento de la calidad y la pertinencia social.

\section{ABSTRACT}

The management of the process of professional training in universities has as via essential a system of methodological work that qualifies directive and educational for the understanding and setting in practice of the plans and study programs, sustained in the pedagogic principle of the unit of the instruction and the education and of linking of the theory with the practice that locates the educational work in the center of the process and its results, with an innovative focus, transformer and prospective. The objective of the report is to reveal qualities that propitiate the understanding of the importance of the methodological work as strategic support of the administration of the process of the professional's training in the universities, of its didactic, systemic, preventive and participative nature to promote the change of the improvement of the quality and the social ownership. 


\section{INTRODUCCIÓN}

Las transformaciones de la educación superior, como políticas públicas, tienen el propósito de mejorar la calidad de la formación integral del profesional, lo que se materializa actualmente en Cuba a través de una nueva generación de planes de estudio sustentada en un modelo que define el futuro profesional capaz de asumir los retos que el desarrollo sostenible del país demanda y que plantea a la universidad nuevas metas, modos de hacer y pensar en la gestión del proceso formativo.

El trabajo metodológico, como proceso, se desarrolla a través de un sistema de actividades con el propósito de capacitar a directivos y docentes para la comprensión, implementación y evaluación del modelo de formación, sus planes y programas de estudio, concepciones curriculares, estrategias educativas, la integración con las instituciones investigativas, de la producción y los servicios; lo que tiene su fundamento en la introducción de resultados de la actividad investigativa, la superación de postgrado y las experiencias de avanzada en el proceso docente educativo de los colectivos pedagógicos.

La gestión del proceso de formación del profesional en las universidades es una categoría que ofrece una comprensión amplia de la acción participativa de todos los integrantes de la comunidad universitaria, en la que prevalece el carácter colectivo más que el individual. La gestión educacional avanza hacia una nueva manera de comprender y dirigir la institución de educación superior en su planificación, organización, control y valoración, de manera que ésta pueda generar otros modos de comunicación y la toma de decisiones cada vez más efectivas.

Una cualidad esencial del trabajo metodológico como proceso es su capacidad transformadora, su concepción estratégica, que proyecta desde el estado actual la gestión para enfrentar los retos y cambios necesarios en los modos de actuación, eliminar las carencias, consolidar fortalezas en función de la eficiencia, eficacia, sostenibilidad y pertinencia de la formación del profesional, asegurar que las universidades den respuesta, con sus egresados, a los objetivos del desarrollo social y económico desde el nivel local hasta el nacional y mundial.

Significa que la relación entre el estado actual, el estado esperado y el posible, enmarca la necesidad creciente de soluciones novedosas a los problemas, que son producto de las investigaciones educativas y las experiencias que los colectivos pedagógicos de las carreras, agrupados en sus años académicos y disciplinas desarrollan, validan y socializan y se convierten en propuestas metodológicas que se amplían y enriquecen hasta convertirse en modelos o estrategias didácticas, metodologías, proyectos o programas, sistemas de acciones o actividades, sistemas de ejercicios o problemas que se transfieren y generalizan en una diversidad de contextos docentes, de la producción y los servicios. 


\section{DESARROLLO}

La Resolución No. 01 /2015, del Ministerio de Educación Superior establece en su Resuelvo Primero, Artículo 5 que los centros de educación superior tienen entre sus principales funciones: dirigir el proceso de formación integral de los estudiantes universitarios, que les garantice una sólida cultura político ideológico y social humanística, así como una elevada competencia profesional...para lograr el desarrollo de estas funciones es vital la gestión emprendedora de directivos, dirigentes estudiantiles y la comunidad universitaria para alcanzar tales propósitos. Los gestores del proceso formativo son los directivos, docentes, estudiantes, representantes de las organizaciones estudiantiles, de las instituciones de la producción y los servicios y los agentes sociales comunitarios que se integran en la labor socioeducativa y extensionista de la carrera. El desarrollo de la gestión con la intención de transformar o mejorar lo que se hace o se quiere hacer, implica enriquecer la acción y hacerla eficiente, porque utiliza mejor los recursos disponibles; es eficaz porque logra los propósitos y fines perseguidos y es pertinente porque es adecuada al contexto y a las personas que la realizan.

Las formas de actuación se pueden construir a partir de la reflexión de los directivos y docentes sobre su propia acción, lo que expresa su autonomía y capacidad de autotransformación; los procesos de formación y aprendizaje y la generación de nuevas herramientas, vías, estrategias que conduzcan con éxito al resultado esperado. La práctica de la gestión educativa, en particular, es consecuencia de las políticas educativas que definen los cambios encaminados al perfeccionamiento de los procesos y sus resultados y cómo se llevan a cada contexto. Según Sañudo (2006) se define como un "proceso mediante el cual se organizan las interacciones afectivas, sociales y académicas de los individuos que son actores de los complejos procesos educativos y que construyen la institución educativa para lograr la formación de los individuos y de los colectivos. La gestión educativa es expresión de la concreción de procesos de planificación estratégica que sustentan el diseño, ejecución y validación de acciones de intervención, organización y comunicación para dirigir la institución, compartiendo la misión, visión, objetivos, resultados esperados y valores entre los miembros de la comunidad educativa, con la definición de los plazos en los que se proyectan las transformaciones en los actores del proceso y en la actividad administrativa y de servicios, así como las relaciones interinstitucionales.

La gestión educativa de la Universidad, en su misión en la red de centros de educación superior, se ocupa de poner en práctica los fundamentos pedagógicos en la dirección del proceso de formación integral de los estudiantes con la implementación de las políticas expresadas en el modelo de formación del profesional que se adecua a un contexto de desarrollo social y económico específico y que demandan, en consecuencia, modos de hacer y pensar propios, lo que se expone a través del proyecto educativo que asegura la conducción por los directivos, docentes y otros agentes de un sistema de acciones que aúnan los esfuerzos en un objetivo común, concertado en espacios de debate y reflexión que contribuyan a delinear el camino hacia la meta. Estas cualidades muestran la gestión educativa como un proceso innovador, transformador y prospectivo. 
La gestión educativa tendrá como prioridades:

1. Preparar al claustro con énfasis en los profesores principales de año académico (PPAA).

2. Consolidar el funcionamiento de los órganos colectivos para la labor educativa en el eslabón de base (colectivos de carreras y años).

3. Estimular el protagonismo y autogestión del aprendizaje de los estudiantes desde la clase, con los recursos existentes.

4. Reforzar el proceso de formación desde la integración de la actividad académica, la investigación, la innovación y la extensión universitaria.

5. Preparar los dirigentes estudiantiles hasta la base.

6. Promover la participación activa en proyectos de orientación educativa hacia prioridades relacionadas con los objetivos del desarrollo sostenible y su implementación en el contexto de la formación de pregrado.

7. Promover la participación en proyectos socioculturales y comunitarios.

8. Fortalecer la orientación profesional desde la relación de la universidad con los sectores de la producción, los servicios, instituciones, asociaciones, etc.

9. Sistematizar los espacios de debates entre profesores y estudiantes y entre los estudiantes.

10. Potenciar el significado de la universidad y su papel en la sociedad contemporánea y el significado de la construcción de una sociedad próspera y sostenible.

11. Atender el mejoramiento de las condiciones de los locales docentes y las residencias estudiantiles.

Lo innovador enriquece permanentemente el modelo de gestión y "significa también actualizar sistemáticamente, en los niveles de grado y posgrado, los planes de estudio, las formas de enseñanza, los métodos de evaluación, las relaciones entre alumnos y profesores; incorporar las tecnologías más avanzadas y pertinentes para los fines educativos, entre otros aspectos.

Lo esencial es que no es un proceso espontáneo, la innovación requiere de contar con las políticas institucionales que promuevan los procesos sustantivos de ahí que se convierte en una herramienta para tomar decisiones, definir prioridades, puntualizar la visión que marca los resultados y los impactos que se esperan y que dinamicen las fuerzas internas de la institución que impulsan los procesos de transformación, en los diversos y cambiantes contextos en que se concretan las acciones con objetivos comunes a toda la comunidad universitaria, pero con la suficiente flexibilidad para incorporar, modificar y reorientar el (o los) camino (s) que conducen a las metas.

Es el trabajo metodológico la vía fundamental para innovar en la búsqueda de soluciones en la preparación óptima para conducir la formación del profesional, ya que apoyado en la Didáctica, es la labor que realizan los sujetos que intervienen en el proceso docente educativo, con el propósito de alcanzar relevantes resultados en dicho proceso, jerarquizando la labor educativa desde la instrucción, para satisfacer plenamente los objetivos formulados en los planes de estudio (Artículo 24 de la RM 210/07). 
Para el adecuado cumplimiento de sus funciones (Artículo 26 de la RM 210/07) que tienen como sustento esencial lo didáctico, se parte de la consideración de que el trabajo metodológico se realiza en los niveles de dirección: departamento docente, facultad, filial o unidad docente, sede universitaria y centro de educación superior; así como en los niveles organizativos: colectivo de carrera, colectivo de disciplina, colectivo de asignatura y colectivo del año académico. Esos niveles trazan estrategias pedagógicas comunes, en correspondencia con su papel y lugar en la estructura del centro de educación superior, dirigidas al perfeccionamiento del proceso docente educativo y divulgarán las mejores experiencias de los colectivos metodológicos en los claustros para su generalización.

La lógica del trabajo metodológico, en todos los colectivos y niveles de dirección, se desarrolla atendiendo a los objetivos generales previstos en el modelo del profesional. Los problemas detectados durante el proceso docente educativo se toman como punto de partida para establecer dichos objetivos, lo cuales se concretan en acciones específicas a desarrollar, utilizando las formas y tipos que se establecen en el RM 210/07 u otras que surjan en la dinámica de este trabajo. Las acciones a realizar se plasman en un plan de trabajo metodológico (PTM) para cada curso académico. Los planes de trabajo metodológico de cada uno de los colectivos y niveles de dirección deben estar elaborados al inicio de cada curso académico y podrán adecuarse para cada período lectivo, en correspondencia con el diagnóstico y los resultados que se vayan alcanzando (Artículo 42 de la RM 210/07). El plan de trabajo metodológico (PTM) es el documento que legitima el trabajo docente y científico metodológicos que se realiza en los niveles de dirección y organizativos del trabajo metodológico y es aprobado por el Rector, Decano, Director de Centro Universitario Municipal (CUM) o Jefe de departamento, para todos los niveles subordinados, quienes, según corresponde, lo firman. Su elaboración:

$>$ Debe tener una adecuada concepción y para ello es necesario:

$\checkmark$ El establecimiento de prioridades partiendo de las más generales hasta las más específicas, que se definen a partir de las debilidades que merecen ser atendidas por vía del trabajo metodológico.

$\checkmark \quad$ El carácter diferenciado y concreto del contenido del trabajo metodológico en función de los problemas y necesidades de cada instancia y grupo de docentes.

$\checkmark \quad$ La combinación racional de los elementos filosóficos, políticos, científico- teóricos y pedagógicos en el contenido del trabajo metodológico.

$\checkmark \quad$ El carácter sistémico, teniendo en cuenta la función rectora de los objetivos, al vincular diferentes niveles organizativos y tipos de actividades.

El carácter en sistema se fundamenta en:

$\checkmark \quad$ La vinculación que existe entre los objetivos y las actividades que se llevan a cabo en cada uno de los niveles que comprenden un subsistema del proceso.

$\checkmark \quad$ La planificación coordinada del trabajo para seleccionar acertadamente los propósitos a lograr por etapas durante todo el curso. 
$\checkmark \quad$ El tratamiento a los problemas metodológicos desde la interrelación de los diversos tipos de actividades metodológicas.

$\checkmark \quad$ La prioridad de las tareas más generales e importantes y en la cantidad lógica que permitan planificarlas y desarrollarlas con efectividad.

> Proyectarse considerando el diagnóstico general e individualizado de cada docente, estudiante y proceso docente educativo; para determinar el contenido concreto a tratar en función de los problemas y necesidades, además del aprovechamiento óptimo de todos los espacios para la demostración, el debate científico, su control y el cumplimiento de los objetivos trazados. Esa proyección se concreta en los planes de trabajo metodológico que se elaboran en todos y cada uno de los niveles de dirección y organizativos:

$\checkmark$ A nivel de departamento carrera o multicarrera, debe tenerse el plan de trabajo metodológico del departamento docente, de la(s) carrera(s), de los colectivos de las disciplinas, de los colectivos de las asignaturas y de los colectivos de año académico.

$\checkmark$ A nivel de departamento disciplina debe tenerse el plan de trabajo metodológico de este, de los colectivos de disciplinas y asignaturas, y si tiene presencia en el CUM del colectivo de asignaturas de los CUM.

$\checkmark \quad$ Los CUM tienen su propio plan de trabajo metodológico a su interior donde contemplen las actividades metodológicas de las carreras, las disciplinas y asignaturas, así como la de los años académicos.

$>$ En cada uno de planes de trabajo metodológicos, los objetivos propuestos deben materializarse mediante actividades metodológicas concretas a desarrollar por cada colectivo, garantizando su correspondencia con las orientaciones dadas por los diferentes niveles de dirección y con las funciones que sobre la labor educativa se norman en la Resolución No. 210/07 (artículos 33, 35, 38 y 40).

$\checkmark$ Las actividades metodológicas deben concebirse en forma de ciclo, a saber: secuencia gradual de actividades docente-metodológicas, desde una concepción sistémica para favorecer el tratamiento al mismo objetivo. En los niveles organizativos de la carrera ese ciclo es: reunión metodológica- clase metodológica - clase abierta taller metodológico. En cada instancia donde se desarrolla esta labor, atendiendo a su composición, se determina el ciclo de actividades a desarrollar.

> El carácter en sistema de los planes de trabajo metodológico en las carreras debe considerar el abordaje con suficiente profundidad metodológica y científica de la interdisciplinariedad a favor de un mayor dominio del modo de actuación profesional, garantizar un trabajo de gestión de la calidad dirigido a la implementación del currículo y a su perfeccionamiento continuo en los niveles subordinados, para lograr la unidad entre la educación y la instrucción, la validación de los programas que integran el Plan del Proceso Docente de la cual se derivan sus modificaciones. 
$>$ En el mes de junio se reciben las orientaciones de los niveles superiores para la elaboración de los planes de trabajo metodológico y se establecen los calendarios de balances del trabajo metodológico desarrollado y la confección de los del próximo curso académico.

$>$ Los planes de trabajo a todos los niveles pueden ser ajustados durante el curso académico, siempre y cuando este ajuste conduzca a la solución de problemas reales presentados. Se elaboran antes del concluir el curso precedente, es decir en el mes de julio de cada curso académico.

El trabajo metodológico es transformador, como proceso es una mirada al futuro, se concibe sobre qué hacer para transformar el estado actual en la formación del profesional (esencialmente desde la relación profesor - estudiante) al estado deseado (desempeño del estudiante), proyecta los posibles cambios por etapas y se basa en la toma de decisiones, principalmente en los colectivos de docentes, de cuáles formas y métodos aseguran que se produzcan esos cambios. Lo prospectivo es concebido como multidisciplina del conocimiento para pensar, debatir y modelar el futuro, que utiliza variados métodos y técnicas de análisis y monitoreo de los cambios presentes. Tiene como objeto el análisis de sistemas sociales con el propósito de conocer mejor la situación presente, identificar tendencias futuras, visualizar escenarios ulteriores y analizar el cambio tecnológico y no tecnológico en la sociedad. La prospectiva se refiere a la sociedad futura a la que se aspira, teniendo en cuenta las condiciones de la actual etapa de la construcción de la sociedad y atañe a la visión prospectiva pensar en las acciones y medidas concretas para alcanzar los objetivos, el ideal de futuro, las principales transformaciones, lo que hay que cambiar con el objetivo de avanzar y consolidar los principios de nuestro socialismo y construir una nación soberana, independiente, socialista, democrática, próspera y sostenible.

La gestión educativa en la Universidad de Oriente a partir de los resultados de los indicadores de eficiencia y las cualidades que definen la formación del profesional ha evidenciado que en este proceso ha prevalecido un análisis fragmentado que limita la visión de la formación integral en la caracterización del producto final y de la naturaleza de esos resultados. El análisis y valoración de las estrategias educativas y de trabajo político ideológico, de cómo se implementa la concepción curricular y se aprecian los resultados e impactos de los planes de trabajo metodológico de los años académicos, disciplinas, carreras, departamentos docentes, facultades, centros universitarios municipales y la universidad en la formación integral de los estudiantes constituyen referentes imprescindibles en la gestión institucional. De ahí que se convierten en problemas conceptuales para el trabajo metodológico en todos los niveles de dirección y colectivos de carreras, disciplinas y años académicos interrogantes como las siguientes:

$\square \quad$ ¿Se logran los niveles esperados en el desempeño de directivos, docentes y estudiantes en la implementación del modelo de formación del profesional?

$\square \quad$ ¿Es la Estrategia Educativa y de Trabajo Político Ideológico un instrumento para dirigir el proceso de formación integral en todos los escenarios? 
$\square \quad$ ¿Se aprovechan racionalmente las potencialidades de todos los escenarios intra y extrauniversitarios en la labor educativa desde la instrucción de los estudiantes?

¿Los resultados del proceso docente se analizan desde una visión integradora de los procesos (académico, laboral, investigativo, extensionista) y del sistema de influencias que ejercen los colectivos de carreras, disciplinas y años académicos y modos de actuación de los estudiantes?

$\square$ ¿Los resultados de los indicadores cuantitativos del proceso docente educativo se analizan y valoran cualitativamente desde el significado político, social y educativo de los conceptos de eficiencia, eficacia y efectividad de la formación del profesional?

El análisis de estos problemas demanda la reflexión acerca de conceptos como: calidad de la formación, formación integral del profesional, formación continua, escenarios educativos, estrategia educativa y de trabajo político ideológico y eficiencia académica, lo que sustentan los fundamentos generales del modelo de formación que perfecciona actualmente la educación superior cubana. La formación integral del profesional en las universidades cubanas es comprendida como un sólido desarrollo académico, científico, amplia cultura científica, ética, jurídica, humanista, económica y medio ambiental; comprometidos y preparados para defender las causas justas de la humanidad con argumentos propios, y competentes para el desempeño profesional y el ejercicio de una ciudadanía virtuosa. Esto tiene su expresión en la capacidad de contribuir, de forma creadora, a encontrar solución a los problemas de la práctica.

La formación continua es el proceso de formación y desarrollo profesional con responsabilidad ética, social y ambiental, que el egresado demuestre una alta calificación en su desempeño profesional y posea cualidades personales que lo ayuden a conjugar sus intereses personales con los de la sociedad y participe activa, crítica y constructivamente en su desarrollo. La formación continua se desarrolla en tres etapas: la formación de pregrado en carreras de perfil amplio, la preparación para el empleo y la formación de postgrado. La formación integral y la continua se llevan a cabo en los escenarios educativos que son todos los espacios en que transcurre el accionar de los estudiantes en la Universidad, en las unidades docentes y otros escenarios comunitarios en que se realizan las actividades que tienen un objetivo en su preparación profesional y para la vida.

\section{El trabajo metodológico}

El sistema de trabajo metodológico asume los problemas conceptuales relacionados con la integralidad de la formación del estudiante en direcciones como las siguientes:

La capacidad de gestionar su aprendizaje haciendo uso racional y eficiente de los recursos existentes, de las técnicas de estudio que le propicien la búsqueda y procesamiento de la información, la presentación y argumentación de los resultados.

$\square \quad$ La participación activa en las actividades curriculares, extensionistas y sociopolíticas, con elevado protagonismo en las tareas de impacto social y económico, capaces de demostrar los cambios y transformaciones que se producen. 
$\square \quad$ La construcción de un proyecto de vida que jerarquice la motivación y compromiso con el ejercicio de la profesión y la contribución a la solución de los problemas de su país, su localidad y la universidad.

$\square \quad$ La demostración de modos de comportamiento que se correspondan con los diversos escenarios y actividades en que participan, que los caracterice la puntualidad, disciplina, cortesía, la comunicación asertiva y pertinente y la crítica oportuna.

$\square$ La expresión oral y escrita capaz de exponer, argumentar, demostrar posiciones, ideas, resultados o soluciones a problemas con elevado dominio de la lengua materna, unido a una adecuada presencia personal en cada contexto.

El trabajo metodológico constituye un modelo de gestión educativa que se encamina a preparar la ruta hacia el futuro deseable y el realmente posible, orientar las acciones alternativas que fomenten en los gestores del proceso de formación del profesional una actitud para favorecer el cambio o transformación e impulse el diseño del futuro. El trabajo metodológico tiene en su esencia gestionar la formación integral del profesional en las universidades y para ello se propone capacitar a directivos y docentes en el diseño, ejecución y evaluación del proyecto educativo institucional y su concreción en cada carrera y año académico, lo que toma como premisas:

La concreción del modelo de formación del profesional en el contexto social y comunitario de la universidad, su visión a mediano y largo plazos en correspondencia con las metas que en el desarrollo económico y social se plantea el país y exige a la educación superior. La unidad instrucción - educación y de la teoría con la práctica en el desarrollo de una cultura laboral, económica, jurídica, ambientalista, etc.

$\square \quad$ La integración de las estrategias que dan cumplimiento a los objetivos de la formación integral del profesional.

$\square \quad$ La contextualización de las exigencias del currículo a las condiciones reales que se determinan del diagnóstico de la comunidad educativa, el desarrollo local y las demandas de los procesos productivos, de servicios, investigativos, etc.

$\square \quad$ La definición de las vías para la intervención educativa por todos los agentes socializadores en la formación del estudiante en todos los escenarios intra y extrauniversitarios.

$\square \quad$ La orientación de las formas de participación protagónica de los estudiantes en todos los procesos y resultados de las actividades docentes, extra docentes y socio políticas.

$\square \quad$ La orientación de la capacitación e innovación de los directivos y personal docente en función de la solución de los problemas diagnosticados y sus causas.

$\square$ El análisis de la infraestructura y su uso óptimo en las áreas docentes, deportivas, administrativas y de servicios y los recursos disponibles para cada proceso.

Son ideas rectoras en la concepción del trabajo metodológico:

La integración de los procesos en función de la misión, objetivos y metas.

La sostenibilidad en las metas, las acciones y resultados.

La sistematicidad en la ejecución de las soluciones con la participación de los docentes 
en la toma de decisiones.

La unidad de lo estratégico y lo táctico - operativo.

El carácter transformador y medible de las soluciones que se proponen.

La innovación para poner en práctica alternativas, experiencias y de las buenas prácticas educativas.

La experiencia en la concepción del trabajo metodológico en los diferentes niveles de dirección de la Universidad y de los colectivos metodológicos en el eslabón de base en las carreras se sistematiza en un procedimiento para el diseño, elaboración e implementación de un plan de trabajo metodológico con enfoque innovador, transformador y prospectivo que implica los pasos siguientes:

Diagnóstico integral de la comunidad educativa del año académico, disciplina, carreras, departamentos docentes, facultad, centro universitario municipal o universidad a partir de los resultados de su desempeño, indicadores de eficiencia del proceso docente educativo, niveles de satisfacción, procesos de mejora de la calidad, pertinencia y sostenibilidad de las prácticas educativas.

$\square$ Identificación de las manifestaciones en el desempeño de directivos, docentes y estudiantes en el proceso de formación integral del profesional, con énfasis en las particularidades en el proceso de enseñanza aprendizaje.

$\square$ Determinación de los problemas conceptuales metodológicos que revelen las carencias, necesidades e insuficiencias en la gestión educativa de directivos y docentes y la valoración de las posibles causas.

$\square$ Determinación y formulación de los objetivos conceptuales metodológicos que delimiten las direcciones, metas o alcances hacia las que se encaminan las propuestas de soluciones.

$\square$ Búsqueda de posibles soluciones en experiencias pedagógicas de los docentes, resultados de las investigaciones o de actividades de superación y socialización, para su contextualización en correspondencia con el diagnóstico integral.

$\square$ Presentación y socialización de la propuesta a través de reuniones o talleres metodológicos, clases metodológicas instructivas y/o demostrativas y abiertas.

$\square \quad$ Toma de decisiones (acuerdos de la actividad metodológica) acerca de la (o las) vía(s) de solución que da(n) respuesta a los problemas identificados, así como los instrumentos o formas de evaluación de los resultados.

$\square \quad$ Introducción en la práctica educativa de cada docente de la (o las) vía(s) de solución, análisis y valoración de los cambios, transformaciones y avances en el proceso formativo.

$\square$ Generalización de experiencias a través de resultados en materiales docentes, medios de enseñanza, propuestas curriculares, métodos de enseñanza, modelos pedagógicos y didácticos, estrategias, metodologías, proyectos educativos, sistemas de acciones, sistemas de ejercicios y problemas, programas de asignaturas y disciplinas, orientaciones metodológicas; conjunto de instrumentos para la medición de la eficacia, eficiencia y factibilidad; planes para la mejora de la calidad, etc.

$\square$ Validación a mediano y largo plazos de la sostenibilidad de los resultados en el mejoramiento continuo de la calidad de los procesos. 
El procedimiento anterior pone énfasis en eliminar barreras en los colectivos de carreras, disciplinas y años académicos relacionados con insuficiencias como las siguientes:

Falta de profundidad en la valoración causal de los problemas que se identifican en los modos de actuación de directivos, docentes y estudiantes; los materiales y medios que se emplean en el proceso de formación del profesional.

$\square \quad$ No siempre se concentran los análisis en el desempeño de directivos y docentes, en sus potencialidades y necesidades para proponer soluciones encaminadas al mejoramiento o cambios en su gestión del proceso formativo.

$\square \quad$ El principio pedagógico de la unidad de la instrucción y la educación adolece de comprensión y materialización al prevalecer actividades metodológicas que atienden más a lo instructivo y organizativo y menos a la labor educativa, de motivación y compromiso de los estudiantes con la profesión y la sociedad.

$\square \quad$ Falta enfoque sistémico en el planteamiento de soluciones al concebir el sistema de actividades metodológicas que no transitan desde la identificación de los problemas, su diagnóstico, propuesta de solución, demostración de las vías de solución, control y valoración de la pertinencia a partir de la correspondencia entre los resultados esperados y los logrados.

$\square \quad$ La falta de acuerdos en las actividades metodológicas producto de la socialización de las experiencias e introducción de resultados de la actividad investigativa que limita la participación colectiva en la toma de decisiones.

$\square \quad$ No se proponen instrumentos, científicamente elaborados, para la medición de los cambios o transformaciones que se producen en la formación del profesional a corto, mediano y largo plazos.

$\square \quad$ No siempre las propuestas de solución se sistematizan en materiales docentes, monografías, artículos científicos que se conviertan en medios imprescindibles para la autopreparación de directivos y docentes.

$\square \quad$ Las propuestas para la introducción acelerada de la tecnología en el proceso de formación del profesional, haciendo un uso óptimo de la infraestructura existente, no se visualiza en la gestión de directivos y docentes en la construcción de modelos educativos que impliquen cambios en todas las formas organizativas del proceso docente, con énfasis en la gestión del conocimiento en toda la comunidad universitaria.

Estas barreras se convierten en referentes de las líneas estratégicas para el trabajo metodológico, problemas conceptuales a los que hay que buscar, pensar y materializar las vías de solución; validar y generalizar experiencias y enriquecer la teoría y práctica pedagógicas con nuevos materiales de consulta para los directivos y docentes.

\section{CONCLUSIÓN}

La comprensión de la necesidad de cambiar, emprender vías de solución a los problemas educativos relacionados con la formación del profesional, demanda aplicar los resultados de la actividad científica y sistematizar experiencias pedagógicas novedosas y transformadoras, que promuevan la participación protagónica de los directivos y docentes en la construcción 
de modelos, estrategias, metodologías, proyectos, programas que enriquecen sistemáticamente la práctica educativa y mejoran la calidad de la gestión desde el eslabón de base en la educación superior. El trabajo metodológico ha de consolidarse como herramienta en la gestión para el cambio y mejoramiento continuo, para pensar, debatir y modelar el futuro de las universidades.

\section{BIBLIOGRAFÍA}

1. Alarcón Ortiz, R.: Universidad innovadora por un desarrollo humano sostenible. Conferencia Inaugural, 10. Congreso Internacional de Educación Superior Universidad 2016. Editorial Félix Varela. La Habana, febrero de 2016.

2. Ferrer, M. y A. Rebollar: Las transformaciones educacionales. En Transformación de la institución educativa. Editorial Pueblo y Educación, 2013.

3. ISBN: 978-959-13- 2628-7 (p. 18 - 33).

4. Ferrer, M., Castillo, M. y Rebollar, A. El trabajo metodológico en la gestión educativa estratégica del proceso de formación del profesional. Memorias Congreso Internacional Pedagogía 2017. La Habana. 2017.

5. Ferrer, M. y otros: Los proyectos educativos, una visión prospectiva de la gestión educacional. Sello Editor Educación Cubana. ISBN 978 - 959 - 18 - 1195 - 0. La Habana. 2017.

6. Mc Pherson Sayú, M. y otros: El trabajo metodológico, vía efectiva para la dirección del proceso docente educativo. Seminario nacional de preparación del curso escolar 2013 - 2014. MINED, La Habana. 2013.

7. Ministerio de Educación: Reglamento del Trabajo Metodológico del Ministerio de Educación. Resolución Ministerial 200/2014. Editorial Pueblo y Educación. La Habana. 2014.

8. Ministerio de Educación Superior: Reglamento Docente y Metodológico (Resolución No 210/2007). Producciones gráficas ENPSES. La Habana. 2007.

9. Ministerio de Educación Superior: Sistema de Evaluación y Acreditación de Instituciones de Educación Superior. Editorial Poligráfica Félix Varela. La Habana. Enero, 2015.

10. Ministerio de Educación Superior: Perfeccionamiento del sistema de gestión del proceso de formación integral de los estudiantes universitarios en el eslabón de base. Editorial Poligráfica Félix Varela. La Habana. Octubre, 2013.

11. PCC. Conceptualización del Modelo Económico y Social Cubano de Desarrollo Socialista. Plan Nacional de Desarrollo Económico y Social hasta 2030: Propuesta de Visión de la Nación, Ejes y Sectores Estratégicos. Tabloide. La Habana. 2016. P. 32

12. Sañudo. L. La Transformación de la gestión educativa. Entre el conflicto y el poder.

http://educacion.jalisco.gob.mx/consulta/educar/16/16Lya.html Consultado abril 2016.

13. Universidad de Oriente: Objetivos de trabajo para el año 2016. Santiago de Cuba, enero de 2016. 